\title{
Complex Chromosome-Positive Acute Myelogenous Leukemia Identified 16 Months following the Completion of Capecitabine Chemotherapy for Early-Stage Colon Cancer
}

\author{
Sureerat Jaruhathai ${ }^{a} \quad$ Uraree Phornvoranunt $^{a} \quad$ Waran Wannasirikul $^{b}$ \\ a Department of Internal Medicine, Police General Hospital, Bangkok, Thailand; bepartment \\ of Internal Medicine, Chaophya Hospital, Bangkok, Thailand
}

\section{Keywords}

Capecitabine · Acute myeloid leukemia - Early-stage colon cancer

\begin{abstract}
Capecitabine is an oral chemotherapy that is used to treat several cancer types, including breast, gastrointestinal, hepatobiliary, and ovarian. The use of antimetabolites in cancer therapy has generally not been associated with leukemogenesis. In this report, we demonstrate a case of capecitabine-related acute myeloid leukemia that was diagnosed 16 months after the completion of treatment for early-stage colon cancer, by a complex chromosome analysis 48,XY,6, del(7)(q22),+8,+13,t(13;17)(q12;p13),t(13,21)(q12;122), +mar [Gazi Med J. 2018 Jan;29(1):57-58]. This is the first report to our knowledge of the development of t-AML in a patient with early-stage colon cancer that was caused by capecitabine. We should use capecitabine with caution. Further studies are essential to investigate capecitabine-triggered leukemogenesis.
\end{abstract}

\section{Introduction}

Colon cancer is one of the most common cancers in both males and females. Antimetabolites such as $5 \mathrm{Fu}$ and capecitabine are both commonly used in the treatment of colorectal cancer [1]. Capecitabine is an oral prodrug of 5FU that disrupts cellular metabolism by inhibiting nucleic acid production. The most common hematologic side effects of antimetabolites 
are anemia, neutropenia, lymphopenia, and thrombocytopenia [2]. Therapy-related acute myeloid leukemia ( $\mathrm{t}$-AML) related to the use of antimetabolites is uncommon and is more associated with alkylating agents and topoisomerase II inhibitors. Here, we discuss a patient who was treated with capecitabine as adjuvant chemotherapy for early colon cancer who developed acute myeloid leukemia.

\section{Case Presentation}

A 59-year-old Thai male with no prior medical history or a family history of cancer presented with intermittent mucous bloody stool. He had no significant weight loss. He underwent a colonoscopy, which was significant for a mass in his descending colon with luminal narrowing. A colonic biopsy done at that time confirmed adenocarcinoma. A left hemicolectomy was done within 2 weeks. The pathology report confirmed moderately differentiated adenocarcinoma pT4N0/13 Mo. He started capecitabine $1,250 \mathrm{mg} / \mathrm{m}^{2} /$ day for 8 cycles for 6 months. The capecitabine dose was reduced to $1,000 \mathrm{mg} / \mathrm{m}^{2} /$ day at the second cycle due to side effect of grade 3 palmar-plantar erythrodysesthesia. After he completed adjuvant chemotherapy, he did well and had a regular follow-up every 3 months. He was still in complete remission; 16 months later, he came to the hospital with a low-grade fever, cough, and fatigue. His complete blood count was significant for pancytopenia, and myeloblasts were observed in his peripheral blood smear. He was diagnosed with bacterial pneumonia and sepsis. We treated him with empiric intravenous meropenem $1 \mathrm{~g}$ every $8 \mathrm{~h}$. His bone marrow study was significant for $20 \%$ myeloblasts, and his chromosome study showed a complex chromosome 48,XY,6,del(7)(q22),+8,+13,t(13;17)(q12;p13),t(13,21)(q12;122),+mar [3]. The patient's hospital course was complicated by severe sepsis, and the patient expired 20 days after he was admitted.

\section{Discussion}

t-AML is rarely reported in relation to the treatment of colon cancer. The prognosis of $\mathrm{t}$-AML caused by cancer treatment is grim. The incidence of $\mathrm{t}-\mathrm{AML}$ is $<1 \%$, and its onset can vary from 1 to 10 years after chemotherapy [4]. A recent report from a Swedish leukemia registry found that treatment at a younger age tends to be highly prognostic of a secondary AML [5]. While there are some genetic features related to t-AML, such as a TP53 mutation, chromosome 5 or 7 abnormalities, and complex cytogenetics, the overall triggering mechanism is unknown [6,7]. Alkylating agents, radiotherapy, topoisomerase drugs, and other antitubulin agents tend to increase the risk of t-AML [7]. The onset of t-AML after treatment with alkylating agents is 4-7 years, and the chromosomal abnormalities of t-AML in these cases are often more complex than those seen in patients with de novo AML. These abnormalities involve chromosome 5 and/or 7 in $70-90 \%$ of patients $[6,8]$. In contrast, the onset of t-AML following treatment with topoisomerase-related drugs is only 2-3 years, and the chromosomal abnormalities seen in these cases are translocations involving chromosome segments 11q23,17q21, and/or 21q22, which involve the $M L L$ gene [8]. Capecitabine is an antimetabolite agent, a precursor molecule that is metabolized to $5 \mathrm{FU}$ by tumor cells through a 3-step enzymatic process [2]. Antimetabolite agents have not been associated with acute leukemia. A literature review included reports of t-AML following various capecitabine regimens that included 5FU and radiation (Table 1). There are also several reports of acute myeloid leukemia following capecitabine monotherapy. A metastatic breast cancer patient developed t-AML 3 months after discontinuing capecitabine therapy. Her

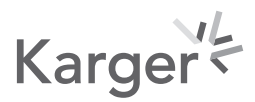


Table 1. Studies of chromosome abnormalities related to AML

\begin{tabular}{lll}
\hline Primary cancer & Treatment & Characteristic \\
\hline Colon cancer stage IV [9] & Capecitabine & t-AML (MLL gene arrangement) \\
\hline Gastric cancer stage IIIc [11] & Oxaliplatin/capecitabine/G-CSF & t-APL \\
\hline Breast cancer stage IV [3] & Capecitabine & t-AML,t(10;11),(q22;q23) \\
\hline $\begin{array}{l}\text { Neuroendocrine tumor } \\
\text { stage IV well differentiated [12] }\end{array}$ & $\begin{array}{l}\text { Capecitabine/temozolemide/ } \\
\text { radiation }\end{array}$ & t-AML (inv(16)) \\
\hline Esophageal cancer stage IV [13] & Cisplatin/5FU/radiation & t-AML (complex karyotype) \\
\hline Colon cancer stage IIB [14] & 5FU/LV bolus & t-AML 46,XY,t(3;21)(q26;q22) \\
\hline \multicolumn{1}{c}{ t-AML, therapy-related acute myeloid leukemia. } & \\
\hline
\end{tabular}

karyotype was 46xx,(10;11)(q22;q23), which is associated with an MLL gene abnormality [3]. Capecitabine-related AML was diagnosed 12 months after the completion of treatment in a patient with metastatic colorectal cancer. $t(6 ; 11)$ with breakpoint 11q23 and MLL gene rearrangement was identified [9]. A patient with rectal cancer developed t-AML 16 months after completing chemoradiation with capecitabine and had a $t(15 ; 17)(q 24 ; q 21)$ translocation in 19 of 20 analyzed cells [10]. In this report, we presented a patient with capecitabinerelated AML and a new cytogenetic anomaly in the setting of early-stage colon cancer. To the best of our knowledge, this is the first report of t-AML with a complex chromosome $48, \mathrm{XY}, 6, \operatorname{del}(7)(\mathrm{q} 22),+8,+13, \mathrm{t}(13 ; 17)(\mathrm{q} 12 ; \mathrm{p} 13), \mathrm{t}(13,21)(\mathrm{q} 12 ; 122),+\operatorname{mar}$ [3]. As we found del 7 in this complex chromosome, this patient's AML may be the result of a converted myelodysplastic syndrome. The prognosis of this pathology is grim despite intensive treatment.

\section{Conclusion}

Prescribers of the antimetabolite capecitabine should be aware of possible leukemogenesis. As capecitabine is a common oral chemotherapy for many kinds of cancer, it is essential that further studies be performed to test its association with AML.

\section{Acknowledgements}

We thank our medical staff who helped and took care of the patient, and we would like to thank Enago for language service.

\section{Statement of Ethics}

Written informed consent was obtained from the family of the deceased patient for publication of this case report.

\section{Conflict of Interest Statement}

The authors have no conflicts of interest to declare.

\section{Karger'}




\section{Case Reports in Oncology}

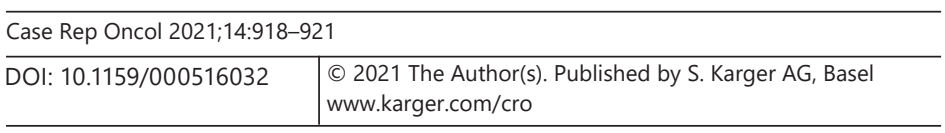

Jaruhathai et al.: Capecitabine Monotherapy-Related Acute Myeloid Leukemia

\section{Funding Sources}

No funding was received for this manuscript.

\section{Author Contributions}

S. Jaruhathai contributed substantially to the concept and drafted the manuscript. U. Phornvoranunt and $\mathrm{V}$. wannasirikul involved in the interpretation of the data, and all authors approved the final version.

\section{References}

1 Cassata A, Procoplo G, Alù M, Ferrari L, Ferrario E, Beretta E, et al. Capecitabine: indications and future perspectives in the treatment of metastatic colorectal and breast cancer. Tumori. 2001 Dec;87(6):364-71.

2 Walko CM, Lindley C. Capecitabine: a review. Clin Ther. 2005 Jan;27(1):23-44.

3 Özkurt NZ, Öcal R, Pepeler SM, Ülküden B. Capecitabine induced therapy related acute myeloblastic leukemia with $\mathrm{t}(10 ; 11)$ (q22;q23) in a patient with breast cancer. Gazi Med J. 2018 Jan;29(1):57-8.

4 Association of chemotherapy for solid tumors with development of therapy-related myelodysplastic syndrome or acute myeloid leukemia in the modern era [Internet]. [cited 2020 May 31]. Available from: https://www. ncbi.nlm.nih.gov/pmc/articles/PMC6439835/.

5 Hulegårdh E, Nilsson C, Lazarevic V, Garelius H, Antunovic P, Derolf ÅR, et al. Characterization and prognostic features of secondary acute myeloid leukemia in a population-based setting: a report from the Swedish acute leukemia registry. Am J Hematol. 2015;90(3):208-14.

6 Zahid MF, Parnes A, Savani BN, Litzow MR, Hashmi SK. Therapy-related myeloid neoplasms: what have we learned so far? World J Stem Cells. 2016;8(8):231.

7 Swerdlow SH, Campo E, Harris NL, Jaffe ES, Pileri SA, Stein H, editors. WHO classification of tumours of haematopoietic and lymphoid tissues. In: WHO classification of tumours. Revised 4th ed. Lyon: International Agency for Research on Cancer; 2017. p. 585.

8 Karp JE, Sarkodee-Adoo CB. Therapy-related acute leukemia. Clin Lab Med. 2000 Mar;20(1):71-81, ix.

9 Shapiro S, Hughes G, Al-Obaidi MJ, O’Reilly E, Ramesh S, Smith J, et al. Acute myeloid leukaemia secondary to treatment with capecitabine for metastatic colorectal cancer. Eur J Haematol. 2007 Jun;78(6):543-4.

10 Sun LM, Lin CL, Lin MC, Liang JA, Kao CH. Radiotherapy- and chemotherapy-induced myelodysplasia syndrome: a nationwide population-based nested case-control study. Medicine. 2015 May; 94(17):e737.

11 Zhang YC. Secondary acute promyelocytic leukemia following chemotherapy for gastric cancer: a case report. World J Gastroenterol. 2015;21(14):4402.

12 Joseph R, McRee AJ, Mathews S, Zeidner JF. Inversion 16 (inv(16)) acute myeloid leukemia (AML) following treatment with radiation, capecitabine, and temozolomide in a patient with metastatic neuroendocrine tumor (NET). Leuk Lymphoma. 2019 Sep 19;60(11):2793-7.

13 Hiraoka S, Sakanaka K, Iwai T, Fujii K, Inoo H, Mizowaki T. Therapy-related acute myeloid leukemia 2 months after chemoradiotherapy for esophageal cancer: a case report. Case Rep Oncol. 2020 Mar;13(1):299-303.

14 Park HJ, Choi JH, Lee KA, Kim HC, Nam YS, Oh YH, et al. A case of therapy-related acute myeloid leukemia following 5-fluorouracil chemotherapy. Korean J Intern Med. 2012;27(1):115. 